\title{
Design, optimization, and evaluation of hydrogel of primaquine loaded nanoemulsion for malaria therapy
}

Pankaj Sharma* (iD) and Mukul Tailang

\begin{abstract}
Background: The present study aimed to design, optimize, and evaluate primaquine loaded nanoemulgel for malaria treatment. Nanoemulgel was prepared with the help of different components such as castor oil, Tween 80: Transcutol P ( $S_{\text {mix }}$ ratio), and polymers. Pseudoternary phase diagram was constructed to optimize $S_{\text {mix }}$ ratio. Response surface methodology was used for the optimization of nanoemulsion preparation based on characterization parameters such as droplet size $(\mathrm{nm})$, zeta potential (mv), polydispersity index (PDI), viscosity $(\mathrm{mPa} \cdot \mathrm{S})$, conductivity $(\mathrm{mS} / \mathrm{cm})$, and percent drug release. Based on these parameter results, F5 formulation was selected as an optimized formulation. F5 formulation was loaded in hydrogel preparation which was developed by using hydroxypropyl methylcellulose (HPMC K15M) 1-2\% concentrations. The prepared nanoemulgel was evaluated with the following parameters: percent drug content, in vitro drug release, ex vivo skin permeation, $\mathrm{pH}$ determination, spreadability determination, and viscosity measurement.

Results: The droplets of primaquine loaded nanoemulsion were nanosized (10-200 nm) in the transmission electron microscope (TEM) images. Zeta potential for all formulations (F1-F9) was observed as $-0.7 \pm 0.02$ to $2.12 \pm$ $0.04 \mathrm{mv}$. Response surface curves were plotted for optimization of perfect nanoemulsion preparation.

Nanoemulgels ( $F 5, F 5_{a}, F 5_{b}$, and F5 $)$ were evaluated for their different parameters such as $\mathrm{pH}\left(F 5,5.2 \pm 0.2 ; F 5_{a}, 5.3\right.$ $\pm 0.1 ; F 5 b, 5.3 \pm 0.1 ;$ and $F 5 c, 5.4 \pm 0.1)$, viscosity (mPa.S) $(F 5,9876 \pm 0.61 ; F 5 a, 14,564.6 \pm 0.42 ; F 5 b, 14,841.9 \pm 0.82$; and $\left.F 5_{c}, 16,872.1 \pm 0.921\right)$, spreadability $(\mathrm{g} . \mathrm{cm} / \mathrm{s})\left(F 5,7.89 \pm 0.10 ; F 5_{a}, 5.09 \pm 0.03 ; F 5_{b}, 4.30 \pm 0.02 ;\right.$ and $F 5_{c}, 3.13 \pm$ 0.01 ), and percent drug content (F5, $100 \pm 0.46 ; F 5_{a}, 98.10 \pm 0.38 ; F 5_{b}, 99.70 \pm 0.41$; and $F 5_{c}, 97.34 \pm 0.51$ ), and ex vivo skin flux of $F_{b}$ was evaluated for $24 \mathrm{~h}$. Ex vivo skin permeability was found $\sim 70 \%$ within $12 \mathrm{~h}$ and $\sim 86 \%$ within $24 \mathrm{~h}$.

Conclusion: The nanoemulsion loaded hydrogel of primaquine with optimum viscosity was prepared for transdermal application. Nanoemulgel was prepared by using HPMC K15M into nanoemulsion because HPMC K15M was responsible for significant viscosity. The permeation rate of nanoemulgel was greater than other drug solutions. The great permeation rate was achieved by the incorporation of Transcutol P (cosurfactant). The optimized formulation was justified by using statistics. Stability studies confirmed that nanoemulgel is a promising carrier for the delivery of primaquine.
\end{abstract}

Keywords: Primaquine, Malaria, Nanoemulsion, Nanoemulgel, Optimization

* Correspondence: pankajsharma223@gmail.com

School of Studies in Pharmaceutical Sciences, Jiwaji University, Gwalior, M.P

-474011 , India 


\section{Background}

Nanoemulsion comes under the category of novel drug delivery systems. It is used to increase the efficacy of a less water-soluble drug once it enters the systemic circulation of a body [1]. Nanoemulsions are formed by the mixture of two immiscible liquids in which one phase is dispersed in another phase. Their size typically ranges from 50 to $200 \mathrm{~nm}$. Mostly, nanoemulsions consist of oil molecules or droplets dispersed in a watery phase or water droplets dispersed in an oily phase depending on their method of preparation. Nanoemulsions show the separation of two different layers within them that is mostly termed as phase separation. Not all of them show phase separation if they show greater stability [2].

The transdermal route for the delivery of drugs in a small dose is very promising. Interest in drug delivery through skin is as well day by day for systemic administration and local effects. There are many significant advantages of the transdermal drug delivery system over other routes of drug delivery, viz. for the avoidance of hepatic first-pass metabolism, gastric irritation, gastric $\mathrm{pH}$, and gastric emptying rate. The hydrogel can also avoid pulsed administration of a drug into the systemic circulation [3]. Although less viscous nanoemulsion restricts its application as transdermal formulation, the rheological behavior of nanoemulsion is modified by gel formation with less interaction of surfactant and cosurfactant [4]. For modification of the rheological behavior of nanoemulsion, various gel matrices such as carrageenan, xanthan gum, and carbomer 940 are used for transdermal formulation [5]. However nanoemulsion loaded hydrogel formulation may be more relevant for transdermal delivery than nanoemulsion.

Primaquine comes under the category of 8aminoquinolone which is a very effective drug in treating malaria. The absorption capacity of primaquine was significantly increased as nanoemulsion formulation $[6,7]$. Primaquine belongs to class III in biopharmaceutical classification with high solubility low permeability $(\log P=$ 2.76). Primaquine shows the first-pass metabolism. However, in the liver, primaquine is metabolized into three metabolites- and 5-hydroxy-6-desmethyl-primaquine, 5hydroxy primaquine, and 8-(3-carboxyl-1-methyl-propylamino)-6-methoxy-quinoline. The major metabolite is carboxyl derivative found in plasma; on repeated administration, it accumulates in the body when unchanged primaquine concentration exceeds than normal limits. These metabolites show less antimalarial effects than primaquine but the hemolytic activity of these metabolites is higher due to the generation of methemoglobin (in vitro) [8-10]. To avoid G.I. disturbances (abdominal pain), nausea, long duration of therapy, bitter taste, high frequency of drug administration, etc., transdermal route was selected for delivery of the drug.
The present study aims to develop novel nanoemulgel of primaquine for better permeability and better applicability through transdermal delivery. Furthermore, constituents of nanoemulsion worked as penetration enhancers thereby avoiding the irritability of chemical permeation enhancers.

\section{Methods}

Primaquine was procured as a gift sample from Indian Pharmaceutical Combine Association Limited laboratories (IPCA laboratories, Ratlam, India). Castor oil I.P. (Indian Pharmacopeia) was purchased from a chemist shop (manufactured by Search Creation Company). Transcutol P (diethylene glycol monoethyl ether), propylene glycol, Span 80, and hydroxypropyl methylcellulose (HPMC) were procured from Himedia Laboratories Pvt. Ltd., Delhi, India. Tween 80 was procured from Ramagundam Fertilizers And Chemicals Limited (RFCL Ltd.) Delhi, India. Solvents, water, and other chemicals were used of analytical grade (chemicals that meet specifications of American Chemical Society (ACS) are called reagent or analytical grade). The animal experimental studies were approved by IAEC (Institutional Animal Ethical Committee) as per the regulation committee for CPCSEA (Committee for Purpose of Control and Supervision of Experiments on Animals) Government of India and registration number of approval was IAEC/JU/47.

\section{Solubility of primaquine}

The solubility of primaquine in castor oil, sesame oil, surfactant (Tween 80, Capryol 90, and Span 80), and cosurfactant (Transcutol P and propylene glycol) was estimated by dissolving excess amount of primaquine in each of selected components (castor oil, surfactant, and cosurfactant) in stoppered vials. Vortex mixer was used for a continuous stirring of drug solution for $20 \mathrm{~min}$ then after it was kept for isothermal shaker for $72 \mathrm{~h}$ at $37^{\circ} \mathrm{C}\left( \pm 0.5^{\circ} \mathrm{C}\right)$. Onto the next step, samples were centrifuged for $20 \mathrm{~min}$ at $3000 \mathrm{rpm}$ and the supernatant was collected and filtered through a membrane filter $(0.45 \mu)$. Then with the help of continuous phase samples were quantified for drug content through UV-VIS spectrophotometer (Shimadzu-1800, Japan) at $259 \mathrm{~nm}$ [11].

\section{Selection of components for nanoemulsion}

Oil was selected based on the maximum solubilization amount of primaquine in different oil. Surfactant and cosurfactant were selected for nanoemulsion formulation on percent transmittance criteria. Emulsification potency of surfactant was determined by adding $200 \mathrm{mg}$ surfactant in $200 \mathrm{mg}$ selected oil. The mixture was homogenized by gentle heating $\left(\sim 40^{\circ} \mathrm{C}\right)$ for $30 \mathrm{~s}$. From this mixture $40 \mathrm{mg}$ was taken and diluted up to $40 \mathrm{ml}$ with water for getting nanoemulsion. Then emulsion was kept 
for $2 \mathrm{~h}$ and analyzed with the help of UV-VIS spectroscopy (Shimadzu-1800, Japan) at $617 \mathrm{~nm}$. Cosurfactant was selected for nanoemulsion formulation in the same manner as the surfactant selection procedure.

\section{Construction of pseudoternary phase diagrams}

Titration technique was used for the construction of the pseudoternary phase diagram of oil, water, surfactant, and cosurfactant [12]. Castor oil was used as an oil phase. $S_{\text {mix }}$ (a mixture of surfactant-Tween 80 and cosurfactant-Transcutol P) was used in four different weight ratios (1:1, 2:1, 3:1, and 4:1) for optimization and determination of best ratio based on the maximum coverage area of nanoemulsion and for optimization and determination of the optimum concentration of oil to $S_{\text {mix }}$ ratio and varied as 9:1, 8:2, 7:3, 6:4, 5:5, 4:6, 3:7, 2:8, and 1:9. Gradually, water was added in a dropwise manner by using microsyringe with vigorous stirring through magnetic stirrer and the endpoint was observed visually for transparency against a dark and light background and experiments were performed in triplicate to get reproducible results. The endpoint of different concentrations of castor oil, $S_{\text {mix }}$ and water were calculated and constructed as triangular coordinates (pseudoternary phase diagrams).

\section{Formulation of nanoemulsion}

Primaquine was dissolved in the mixture of oil, $S_{\text {mix }}$ with different ratio groups from constructed phase diagram as shown in Table 1, and after that water was added gradually in the appropriate quantity. The primaquine containing nanoemulsion was formulated by stirring and vortexing at optimum temperature. All formulations were kept at ambient temperature for onwards studies.

\section{Optimization of nanoemulsion by response variables effects ( $3^{2}$ factorial designs)}

The full $3^{2}$ factorial designs were used for the determination of oil and $S_{\text {mix }}$ concentration effects on different parameters, where oil and $S_{\text {mix }}$ were two variables at three levels (oil 5, 10, and 15 and $S_{\text {mix }} 35,40$, and $45 \%$ $\mathrm{w} / \mathrm{w})$. The prepared nanoemulsion was assessed for responses and the experimental data was collected for comparative study. Based on the comparative study of zeta potential, polydispersity index (PDI), droplet size, and percent drug release, optimum formulation was selected for hydrogel preparation.

\section{Morphological and structural behavior of nanoemulsion}

The morphological and structural behavior of drugcontaining nanoemulsion preparations was assessed with the help of a transmission electron microscope (TEM) (JOEL, USA). Nanoemulsion preparations were diluted with aqua. Then after, a drop of it was directly placed on the leaky porous film grid, phosphotungustic acid solution (1\% aqueous) was used for staining, and droplets size and shape were observed.

\section{Micromeritic properties of nanoemulsion}

The droplet size and PDI of nanoemulsion were measured by Zetasizer (Horiba Scientific SZ-100, Japan) by dynamic light scattering technique. Before the estimation, samples were sonicated and vortexed for getting optimum results.

\section{Conductivity analysis of nanoemulsion}

This test confirmed that the preparation was o/w nanoemulsion. All aqueous solutions have electrical conductance behavior. The measure of a solution those able to conduct electricity is called "conductance" and is the reciprocal of resistance. A conductivity instrument (Aster Conductivity Meter CT-650) measures conductance in which electrodes attached to a sensor dipped in a solution. The analyzer circuitry impresses changes as the voltage on the sensor and records the size of the output signals, which is linearly shown as conductivity.

\section{$\mathrm{pH}$ and viscosity determination of nanoemulsion}

The viscosities of nanoemulsion samples were measured by using a viscometer (Brookfield Ametek, DVE, USA). The 2 number spindle was used in viscometer and dipped in nanoemulsion then rotated at 5, 10, 20, and

Table 1 Solubility of primaquine in various components at $\sim 37^{\circ} \mathrm{C}$ and percent transmittance for various surfactant and cosurfactant

\begin{tabular}{llll}
\hline S. No. & Components & Solubility $\mathbf{( m g / m l )}$ & Percentage transmittance $(\%)$ \\
\hline Oil 1 & Castor oil & $37.32 \pm 0.52$ & - \\
Oil 2 & Sesame oil & $03.32 \pm 0.23$ & - \\
Surfactant 1 & Tween 80 & $52.21 \pm 0.62$ & $97.36 \pm 0.32$ \\
Surfactant 2 & Span 80 & $36.81 \pm 0.46$ & $86.45 \pm 0.56$ \\
Surfactant 3 & Capryol 90 & $34.12 \pm 0.26$ & $66.58 \pm 0.41$ \\
Cosurfactant 1 & Transcutol P & $48.13 \pm 0.45$ & $97.22 \pm 0.37$ \\
Cosurfactant 2 & Propylene glycol & $38.13 \pm 0.19$ & $90.49 \pm 0.60$ \\
\hline
\end{tabular}

Values are shown as mean \pm SD $(=3)$ 
$50 \mathrm{rpm}$ at $25^{\circ} \mathrm{C}$ [13]. At every speed, readings were noted on viscometer. The samples were repeated in the triplicate manner and mean values were calculated.

The digital $\mathrm{pH}$ meter was used for the determination of the $\mathrm{pH}$ of nanoemulsion. Nanoemulsion was weighed $(2 \mathrm{~g})$ and the dispersion medium was purified water $(20$ $\mathrm{ml})$. The 4, 7, and $9 \mathrm{pH}$ buffer solution was used for calibration of $\mathrm{pH}$ meter. The samples were repeated in the triplicate manner and mean values were calculated [14].

\section{Hydrogel preparation by using nanoemulsion}

All formulation of nanoemulsion (F1-F9) was sized in nanometer range and optimized formulation (F5) was integrated with the gel matrix (hydrogel). The HPMC $\mathrm{K} 15 \mathrm{M}$ was used as a gelling agent and hydrogel was used for improving the viscosity of nanoemulsion. The HPMC $\mathrm{K} 15 \mathrm{M}$ was kept for $24 \mathrm{~h}$ in a little amount of water for swelling so that viscous solution can be obtained. There were used different $(1 \%, 1.5 \%$, and $2 \% \mathrm{w} / \mathrm{w})$ concentrations of polymer to obtain optimum viscosity of hydrogel and the final concentration of hydrogel components was decided based on transparency and viscosity $[15,16]$.

\section{Characterization of nanoemulsion loaded hydrogel Determination of drug content}

The drug content in the formulated nanoemulgel was measured by adding $150 \mathrm{mg}$ of formulated nanoemulgel in $15 \mathrm{ml}$ distilled water. This nanoemulgel mixture was used for the determination of drug content through UV spectrophotometer at $259 \mathrm{~nm}$ and distilled water was used as a reference.

\section{The $\mathrm{pH}$ determination}

The digital $\mathrm{pH}$ meter was used for the determination of the $\mathrm{pH}$ of nanoemulsion loaded hydrogel. Nanoemulgel was weighed $(2 \mathrm{~g})$ and the dispersion medium was purified water $(20 \mathrm{ml})$. The 4,7 , and $9 \mathrm{pH}$ buffer solution was used for calibration of $\mathrm{pH}$ meter. The samples were repeated in the triplicate manner and mean values were calculated [14].

\section{Spreadability determination}

The formulated nanoemulgel spreadability was analyzed after $72 \mathrm{~h}$ of the formulation by determining the spreading diameter of preparation between the two glass plates after $1 \mathrm{~min}$. A 400-mg nanoemulgel was weighed and placed on a $1-\mathrm{cm}$ premarked circle on a glass plate and the second plate was placed on over it when weight was increased on the upper plate, and increased diameter of the gel was noted and calculated by using the following equation:

$$
S=\frac{m \cdot l}{t}
$$

where $S=$ spreadability of nanoemulgel, $m=$ weight placed on the upper glass plate, $l=$ length upper of the glass plate, and $t=$ time taken.

\section{Viscosity measurements and rheological behavior}

The viscosity of optimized nanoemulsion (F5) was observed as $22.4 \pm 0.31(\mathrm{mPa} \cdot \mathrm{S})$ as shown in Table 2 . The viscosity of different nanoemulgels having various polymer quantities is shown in Table 3. As the viscosity of HPMC K15M (1.5\%) was optimum among other concentrations (1\% and $2 \%$ ), $1.5 \%$ of HPMC $\mathrm{K} 15 \mathrm{M}$ was found as a suitable one. The viscosity of different nanoemulsion loaded hydrogel is shown in Table 4.

\section{In vitro drug release studies}

Franz diffusion cell with cellophane membrane was used for in vitro drug release studies of nanoemulsion loaded hydrogel. The total capacity of the water-jacketed compartment was $50 \mathrm{ml}$ and it had two arms one for sampling and another for a thermometer. The internal diameter of the donor compartment was $2 \mathrm{~cm}$ [17]. The donor compartment was placed on the over receptor

Table 2 Characterization of primaquine loaded nanoemulsion

\begin{tabular}{|c|c|c|c|c|c|c|}
\hline Formulation & Droplet size (nm) & Zeta potential (mv) & PDI & Viscosity (mPa.S) & Conductivity (mS/cm) & Drug release $(\%)$ \\
\hline F1 & $97.91 \pm 1.20$ & $-0.91 \pm 0.03$ & $0.162 \pm 0.01$ & $11.8 \pm 0.21$ & $78.31 \pm 0.24$ & $88.60 \pm 1.2$ \\
\hline $\mathrm{F} 2$ & $101.30 \pm 1.0$ & $1.8 \pm 0.11$ & $0.154 \pm 0.04$ & $10.3 \pm 0.30$ & $112.1 \pm 0.21$ & $78.91 \pm 1.1$ \\
\hline F3 & $108.63 \pm 0.20$ & $1.9 \pm 0.12$ & $0.187 \pm 0.05$ & $17.6 \pm 0.11$ & $102.4 \pm 0.41$ & $81.34 \pm 1.3$ \\
\hline $\mathrm{F} 4$ & $40.22 \pm 0.69$ & $-1.81 \pm 0.06$ & $0.167 \pm 0.02$ & $19.8 \pm 0.23$ & $100.7 \pm 0.36$ & $86.23 \pm 0.9$ \\
\hline F5 & $68.5 \pm 0.30$ & $-0.7 \pm 0.02$ & $0.171 \pm 0.03$ & $22.4 \pm 0.31$ & $126.6 \pm 0.34$ & $91.28 \pm 1.1$ \\
\hline F6 & $74.10 \pm 0.51$ & $2.1 \pm 0.09$ & $0.179 \pm 0.05$ & $18.8 \pm 0.46$ & $56.76 \pm 0.23$ & $86.13 \pm 1.6$ \\
\hline F7 & $70.83 \pm 0.68$ & $-0.89 \pm 0.05$ & $0.161 \pm 0.06$ & $27.4 \pm 0.25$ & $90.45 \pm 0.26$ & $87.56 \pm 0.8$ \\
\hline F8 & $39.34 \pm 0.36$ & $-0.99 \pm 0.08$ & $0.180 \pm 0.04$ & $28.5 \pm 0.33$ & $101.9 \pm 0.43$ & $82.88 \pm 1.2$ \\
\hline F9 & $50.12 \pm 1.20$ & $2.12 \pm 0.04$ & $0.175 \pm 0.05$ & $29.8 \pm 0.21$ & $121.5 \pm 0.31$ & $88.91 \pm 1.4$ \\
\hline
\end{tabular}

Values are shown as mean \pm SD $(=3)$ 
Table 3 Composition of nanoemulgel formulations (\% W/W)

\begin{tabular}{lllll}
\hline Components (\% w/w) & F5 & $\mathbf{F 5}_{\mathbf{a}}$ & $\mathbf{F 5}_{\mathbf{b}}$ & $\mathbf{F 5}_{\mathbf{c}}$ \\
\hline Primaquine & 2 & 2 & 2 & 2 \\
Castor oil & 10 & 10 & 10 & 10 \\
$\mathrm{~S}_{\text {mix }}(1: 1)$ & 40 & 40 & 40 & 40 \\
$\mathrm{H}_{2} \mathrm{O}$ & 48 & 48 & 48 & 48 \\
In HPMC K15M & - & 1 & 1.5 & 2 \\
\hline
\end{tabular}

$\mathrm{F} 5, \mathrm{~F} 5_{a}, \mathrm{~F} 5_{b}$, and $\mathrm{F} 5_{c}=$ different concentrations of polymer (HPMC K15M)

compartment. The receptor compartment was filled with phosphate buffer saline and its temperature was $37^{\circ} \mathrm{C} \pm$ $0.5^{\circ} \mathrm{C}$. The cellophane membrane was equilibrated before the application of nanoemulgel equivalent to $15 \mathrm{mg}$ of primaquine onto the donor side. Samples were withdrawn at a fixed time interval through the sampling arm of the receptor compartment, at the same time the same amount of fresh phosphate buffer saline $\mathrm{pH} 7.4$ was replaced and analyzed by using a UV spectrophotometer at $259 \mathrm{~nm}$.

\section{Ex vivo skin permeation study}

Franz diffusion cell was used for ex vivo skin permeation study with an effective diffusion area of $4 \mathrm{~cm}^{2}$. The excised Wistar rat dorsal side skin sample was placed and tied between donor and receptor part of Franz diffusion cell and stratum corneum side of skin towards the donor compartment. Then $15 \mathrm{mg}$ contained nanoemulgel was applied on the donor part of the cell. The PBS 7.4 pH solution was filled in the receptor compartment and the temperature was maintained at $37^{\circ} \mathrm{C} \pm 0.5^{\circ} \mathrm{C}$. At the fixed time interval, the samples were withdrawn and the same quantities of fresh PBS solutions were replaced through sample arm [18]. After that, all samples were filtered and assayed by UV spectrophotometer at $259 \mathrm{~nm}$. Drug permeability (apparent permeability) was calculated by using the following formula:

$$
\begin{aligned}
\text { Apparent permeability } & =\left(\frac{V_{A}}{(\text { area } \times \text { time })}\right) \\
& \times\left(\frac{\text { Drug in acceptor compartment }}{\text { Drug in donnor compartment }}\right)
\end{aligned}
$$

where $V_{A}=$ volume in acceptor compartment, area $=$ surface area of excised skin, and time = total transport time.

\section{Thermodynamic stability of nanoemulsion}

At the particular concentration of each component (oil, $S_{\text {mix }}$, and water) of nanoemulsion, it must be thermodynamically stable without creaming, phase separation, and cracking. Thermodynamic stability of formulated nanoemulsion loaded hydrogel was assessed based on centrifugation and freeze-thaw cycles [19]. The strength of the interfacial film was reflected through centrifugation. The nanoemulgel preparations were centrifuged at $3500 \mathrm{rpm}$ for $30 \mathrm{~min}$. The freeze-thaw cycle was performed as-nanoemulgel was filled in test tubes and then tubes were sealed hermetically and vertically kept for $16 \mathrm{~h}$ at $-21^{\circ} \mathrm{C}$ in the freezer and that after tubes were kept at room temperature $\left(25^{\circ} \mathrm{C}\right)$ for $8 \mathrm{~h}$. Freezethaw cycle was performed three times for getting reproducible results, and the last nanoemulsion loaded hydrogel was assessed for any changes.

\section{Statistical analysis}

One way analysis of variance (ANOVA) was used for statistical analysis of data collected from evaluated formulations [20].

\section{Results}

\section{Selection of nanoemulsion components}

To formulate a primaquine loaded nanoemulsion for transdermal application, it should contain good solubility constituents in the system because the soluble drug can permeate through the skin. The solubility of primaquine in castor oil, sesame oil, surfactant, and cosurfactant was estimated as shown in Table 1. Primaquine has higher solubility in castor oil $(37.32 \pm 0.52 \mathrm{mg} / \mathrm{ml})$ than sesame oil $(03.32 \pm 0.23 \mathrm{mg} / \mathrm{ml})$. Drug solubility in different surfactants-Tween 80, Span 80, and Capryol 90-was $52.21 \pm 0.62,36.81 \pm 0.46$, and $34.12 \pm 0.26 \mathrm{mg} / \mathrm{ml}$ respectively. Drug solubility in cosurfactant-Transcutol P and propylene glycol-was $48.13 \pm 0.45$ and $38.13 \pm$ $0.19 \mathrm{mg} / \mathrm{ml}$ respectively.

So castor oil was selected as oil phase based on solubility data. The surfactant and cosurfactant were selected on the criteria of percent transmittance. Percent transmittance for various surfactants was $97.36 \pm 0.32$ and $86.45 \pm 0.56$ for Tween 80 and Span 80 , respectively. Percent transmittance for various cosurfactants was $97.22 \pm 0.37,90.49 \pm 0.60$, and $66.58 \pm 0.41$ for

Table 4 The $\mathrm{pH}$, viscosity, and spreadability of nanoemulgel formulations (\% w/w)

\begin{tabular}{lllll}
\hline Parameters & F5 & F5 $_{\mathbf{a}}$ & $\mathbf{F 5}_{\mathbf{b}}$ & \\
\hline $\mathrm{pH}$ & $5.2 \pm 0.2$ & $5.3 \pm 0.1$ & $5.3 \pm 0.1$ & $\mathbf{F 5}_{\mathbf{c}}$ \\
Viscosity (mPa.S) & $9876 \pm 0.61$ & $14,564.6 \pm 0.42$ & $14,841.9 \pm 0.82$ & $16,872.1 \pm 0.92$ \\
Spreadability (g.cm/s) & $7.89 \pm 0.10$ & $5.09 \pm 0.03$ & $4.30 \pm 0.02$ & $3.13 \pm 0.01$ \\
Drug content (\%) & $100 \pm 0.46$ & $98.10 \pm 0.38$ & $99.70 \pm 0.41$ & $97.34 \pm 0.51$ \\
\hline
\end{tabular}

Values are shown as mean \pm SD $(=3)$ 
Transcutol P, propylene glycol, and Capryol 90 respectively (Table 1). Based on percent transmittance, Tween 80 was selected as surfactant and Transcutol $\mathrm{P}$ as cosurfactant.

\section{Optimization of $S_{\text {mix }}$ ratio by pseudoternary phase diagram} The nanoemulsion existence was stabilized by the optimum ratio of surfactant and cosurfactant through the construction of the pseudoternary phase diagram. The transparent nanoemulsion region was observed in the ternary phase diagram. The rest of the area in the pseudoternary plot was turbid when it was observed visually [12]. Pseudoternary phase diagrams were plotted separately for every ratio of surfactant-cosurfactant, and based on these diagrams (Fig. 1a), nanoemulsion region was identified as well as optimized. In Fig. 1 a, 1:1 $S_{\text {mix }}$ ratio was used and it observed a significant nanoemulsion region. Based on this result, $S_{\text {mix }}$ ratio $1: 1$ was loaded in the hydrogel. In Fig. 1b, 2:1 $S_{\text {mix }}$ ratio showed higher covered region as compared to Fig. 1a, but higher concentration of surfactant may produce skin irritancy. In Fig. $1 \mathrm{c}$ and d, 3:1 and 4:1 $S_{\text {mix }}$ ratio showed lesser covered region as compared to Fig. 1a. Although $S_{\text {mix }} 1$ : 1 was selected as an optimized ratio, its different concentrations were used in nanoemulsion preparations (Table 5).

\section{Optimization of primaquine loaded nanoemulsion by $3^{2}$ factorial designs}

The purpose of the $3^{2}$ factorial design $\left(3^{2}-2\right.$ variables at three levels) was to select the stages of different independent variables (Table 6) of castor oil $\left(R_{1}\right)$ and $S_{\text {mix }}$ ratio $\left(R_{2}\right)$ with droplet size, PDI, and zeta potential.

Nanoemulsion preparations were optimized by $3^{2}$ factorial designs and on the basis of response surface methodology and other evaluation parameters of nanoemulsions (Table 2); F5 formulation was selected to load in hydrogel.

\section{Fitting of data to the model}

All the responses of nanoemulsion were observed for nine formulations and fitted to various models by the application of response surface methodology (Design-Expert software). In response surface methodology, linearity was observed in the best-fitted model (Fig. 2a-d).

\section{Characterization of nanoemulsion Morphological and structural characterization}

The smaller size of the nanoemulsion droplets is more conducive to increase the absorption of the drug in vivo.
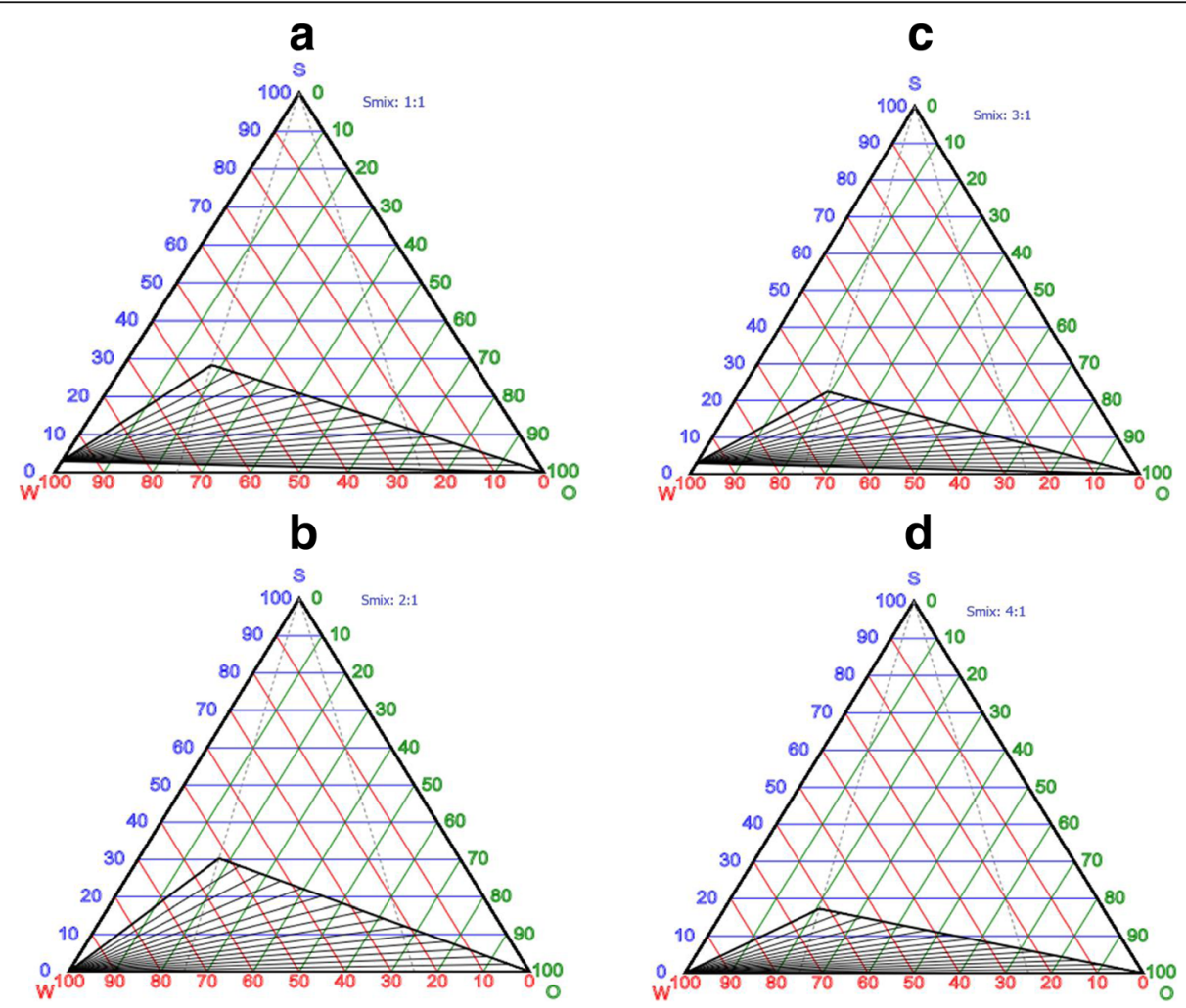

Fig. 1 a The pseudoternary phase plot of $S_{\text {mix }}$ (Tween 80:Transcutol P) at 1:1 ratio. b The pseudoternary phase plot of $S_{\text {mix }}$ (Tween 80:Transcutol P) at 2:1 ratio. c The pseudoternary phase plot of $S_{\text {mix }}$ (Tween 80:Transcutol P) at 3:1 ratio. $\mathbf{d}$ The pseudoternary phase plot of $S_{\text {mix }}$ (Tween 80:Transcutol P) at 4:1 ratio 
Table 5 Composition of primaquine loaded nanoemulsion

\begin{tabular}{lllll}
\hline \multirow{2}{*}{$\begin{array}{l}\text { Formulation } \\
\text { code }\end{array}$} & \multicolumn{4}{l}{ Components $(\% \mathbf{w} / \mathbf{w})$} \\
\cline { 2 - 5 } & Primaquine & Oil $\left(\boldsymbol{R}_{\mathbf{1}}\right)$ & $\boldsymbol{S}_{\text {mix }}(\mathbf{1}: \mathbf{1})\left(\boldsymbol{R}_{\mathbf{2}}\right)$ & Water \\
\hline F1 & 2 & $15(1)$ & $35(-1)$ & 48 \\
F2 & 2 & $15(1)$ & $40(0)$ & 43 \\
F3 & 2 & $15(1)$ & $45(1)$ & 38 \\
F4 & 2 & $10(0)$ & $35(-1)$ & 53 \\
F5 & 2 & $10(0)$ & $40(0)$ & 48 \\
F6 & 2 & $10(0)$ & $45(1)$ & 43 \\
F7 & 2 & $5(-1)$ & $35(-1)$ & 58 \\
F8 & 2 & $5(-1)$ & $40(0)$ & 53 \\
F9 & 2 & $5(-1)$ & $45(1)$ & 48 \\
\hline
\end{tabular}

By analyzing the TEM image of nanoemulsion, it could be revealed that nanoemulsion has a good morphological appearance, uniformed dispersion, and small droplet size, and these images (Figs. 3 and 4) assured that the droplet sizes were in the nano range $(<200 \mathrm{~nm})$.

\section{Micromeritic properties}

The droplet size of nanoemulsion is an essential part of the safe and efficient delivery of dosage form. As the castor oil concentration was kept 5\% w/w, and then droplet size was $39.34 \pm 0.36 \mathrm{~nm}$ observed lowest. As oil concentration was enhanced to $15 \% \mathrm{w} / \mathrm{w}$, the droplet size increased up to $108.63 \pm 0.20 \mathrm{~nm}$ substantially. All preparations were observed in the nanosize range which was expressed by the lower value of PDI. PDI is a ratio of the standard deviation to average droplet size and it shows the uniformity of droplet size within the preparations [21, 22]. The PDI values were low as 0.154 to 0.187 and it indicates a narrow distribution of droplet size within the preparation.

\section{Viscosity and conductivity analysis}

The viscosity of nanoemulsion preparations was determined as $10.3 \pm 0.30$ to $29.8 \pm 0.21 \mathrm{mPa} S$ and was not fit for transdermal application which signifies its viscous gel matrix preparation. The conductivity of formulations was

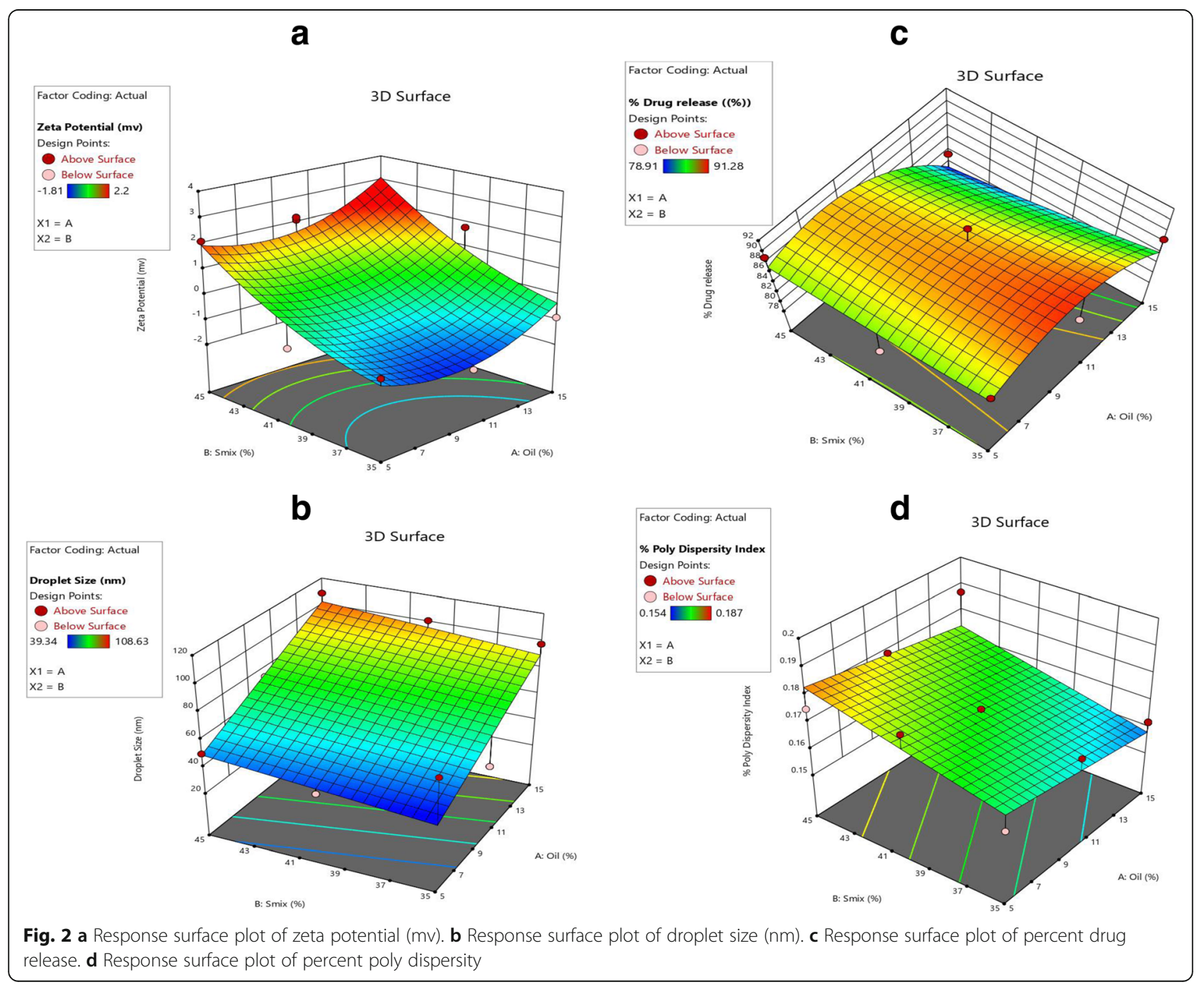


Table 6 Actual unit with coded labels ( $3^{2}$ factorial designs)

\begin{tabular}{llll}
\hline Variables & $\begin{array}{l}\text { Level 1 } \\
\text { (high) }\end{array}$ & Level 0 (medium) & Level 1 (low) \\
\hline Oil Concentration $\left(R_{1}\right)$ & 15 & 10 & 5 \\
$S_{\text {mix }}$ ratio $\left(R_{2}\right)$ & 45 & 40 & 35 \\
\hline
\end{tabular}

found as $56.76 \pm 0.23$ to $126.6 \pm 0.34 \mathrm{mS} / \mathrm{cm}$ which showed that emulsion was o/w type as shown in Table 2.

\section{Percent drug release}

The maximum drug release $(91.28 \pm 1.1)$ was observed within $8 \mathrm{~h}$ for $\mathrm{F} 5$ formulation. The release rate of primaquine loaded nanoemulsion from F5 preparation is shown in Table 2.

\section{Preparation and optimization of primaquine containing nanoemulgel}

For transdermal delivery, primaquine was loaded into a nanoemulgel system having HPMC K15M (1-2\% w/w) as a gel matrix. The optimized viscous system is suitable for transdermal delivery based on biophysical and sensorial evaluations [23]. In the case of HPMC K15M, 1\% $\mathrm{w} / \mathrm{w}$ was added directly to prepare nanoemulsion, and it showed that it took more time for swollen than water. Even some tiny agglomerates of HPMC K15M (1\% w/w) were observed. In the HPMC K15M $(1.5 \% \mathrm{w} / \mathrm{w})$ case, it was swollen in the water phase and its $\mathrm{pH}$ and viscosity were optimum with good homogenization property. In the last case, HPMC K15M ( $2 \% \mathrm{w} / \mathrm{w})$ case, it showed higher viscosity than previous ratios (Table 3 ).

\section{The $\mathrm{pH}$ and viscosity of optimized nanoemulsion (F5) loaded nanoemulgel}

The $\mathrm{pH}$ of nanoemulgel was observed $5.3 \pm 0.1$ for all ratios of polymer, which was closer to skin $\mathrm{pH}$ as shown in Table 4 . The viscosity of $\mathrm{F} 5 \mathrm{a}, \mathrm{F} 5 \mathrm{~b}$, and $\mathrm{F} 5 \mathrm{c}$ were observed 14,564.6 $\pm 0.42,14,841.9 \pm 0.82$, and 14,841.9 \pm 0.82 respectively (Table 4$)$. It was observed that $F 5_{b}$

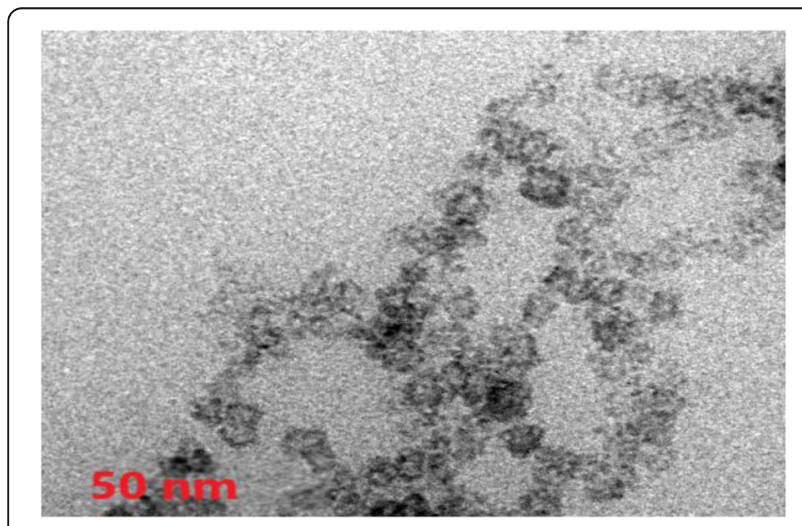

Fig. 3 TEM image of primaquine loaded nanoemulsion contains $1.5 \%$ HPMC K15M showed optimum viscosity in all three formulations.

\section{Spreadability of nanoemulgel}

Spreadability of emulgel formulations was found to be $5.09 \pm 0.03,4.30 \pm 0.02$, and $3.13 \pm 0.01$ g.cm $/ \mathrm{s}$ for $\mathrm{F} 5 \mathrm{a}$, $\mathrm{F} 5 \mathrm{~b}$, and $\mathrm{F} 5 \mathrm{c}$ respectively (Table 4 ).

\section{Determination of drug content}

The content of the drug in various nanoemulgel preparations was found in range $98.10 \pm 0.38$ to $97.34 \pm$ $0.51 \%$, although $100 \%$ of drug content was observed in F5 nanoemulsion preparation. The data of drug content (Table 4) showed that the drug was distributed uniformly throughout the nanoemulgel and the drug loss during or after the nanoemulgel formulation; it was minimum.

\section{In vitro drug release studies}

The percent drug release data show the sustained release of primaquine from the optimized nanoemulgel formulations. Optimized nanoemulsion (F5) and nanoemulgel formulations $\left(\mathrm{F} 5_{\mathrm{a}}, \mathrm{F} 5_{\mathrm{b}}\right.$, and $\left.\mathrm{F} 5_{\mathrm{c}}\right)$ were evaluated for drug release study for $24 \mathrm{~h}$ (Fig. 5).

\section{Ex vivo skin flux and permeation study}

Ex vivo skin flux was determined only for $\mathrm{F} 5$ b formulated emulgel (optimized emulgel) for $24 \mathrm{~h}$. Ex vivo skin flux study was conducted at 1, 2, 4, 6, 8, 10, 12, 18, and $24 \mathrm{~h}$ and flux was observed as $0.49 \pm 0.06,0.45 \pm 0.08$, $0.43 \pm 0.10,0.41 \pm 0.03,0.40 \pm 0.09,0.39 \pm 0.07,0.39 \pm$ $0.08,0.39 \pm 0.01,0.39 \pm 0.03$ respectively. Ex vivo skin permeability was found $\sim 70 \%$ within $12 \mathrm{~h}$ and $\sim 85 \%$ within $24 \mathrm{~h}$ (Fig. 6).

\section{Release kinetic study of emulgel preparations}

Optimized nanoemulgel $\left(\mathrm{F} 5_{\mathrm{b}}\right)$ release kinetic mechanism was analyzed through the data fitting to the Peppas model and Higuchi's model and data compared to the correlation coefficient $\left(R^{2}\right)$. These correlation coefficients $\left(R^{2}\right)$ were observed for zero order, first order, Higuchi's model, Hixon-Crowell model, and Korsmeyer-Peppas model as $0.996,0.853,0.942,0.919$, and 0.979 respectively. The correlation coefficient $\left(R^{2}\right)$ was observed 0.996 for zero order which was higher than other models. So it was obvious that the release of drug from the formulation followed the zero-order release.

\section{Thermodynamic stability of nanoemulsion}

Stability study of optimized emulgel formulation $\left(\mathrm{F} 5_{\mathrm{b}}\right)$ was conducted for 3 months at different temperate conditions $\left(4 \pm 0.2{ }^{\circ} \mathrm{C}, 25 \pm 2{ }^{\circ} \mathrm{C}\right.$, and $\left.40 \pm 0.1^{\circ} \mathrm{C}\right)$. All parameters of optimized emulgel preparation were found in the range at all temperate conditions and these were 
a

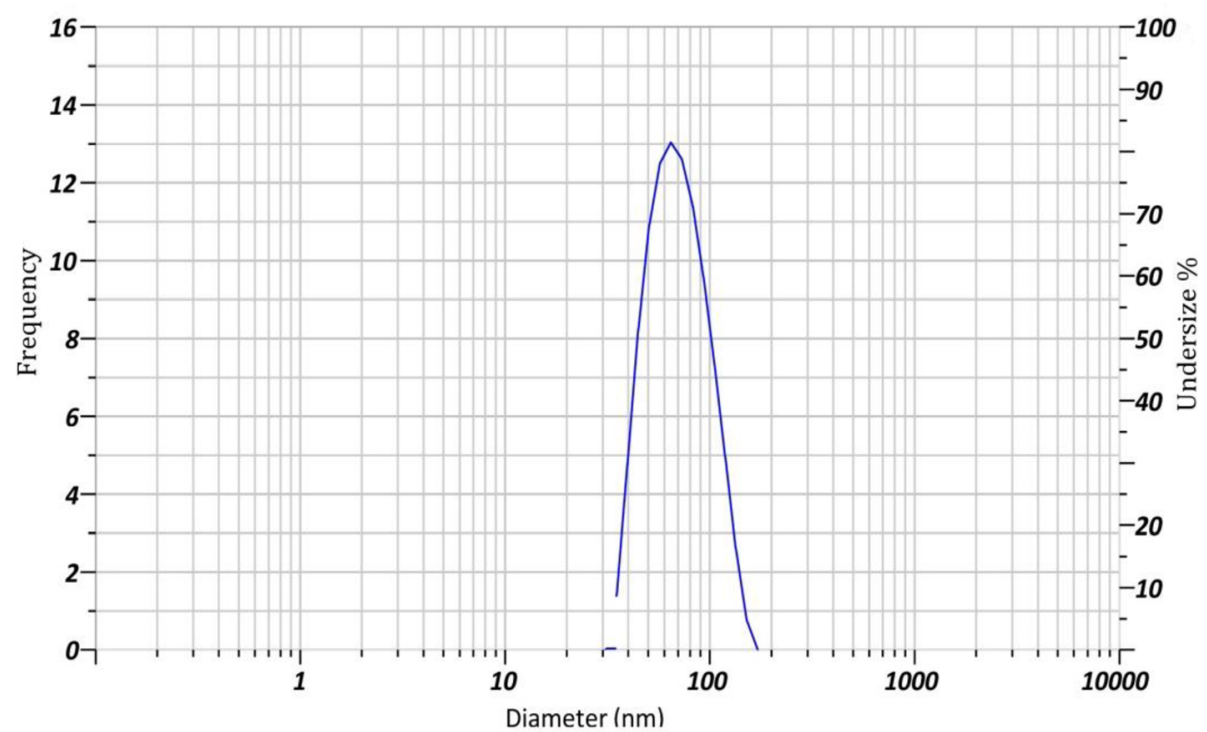

b

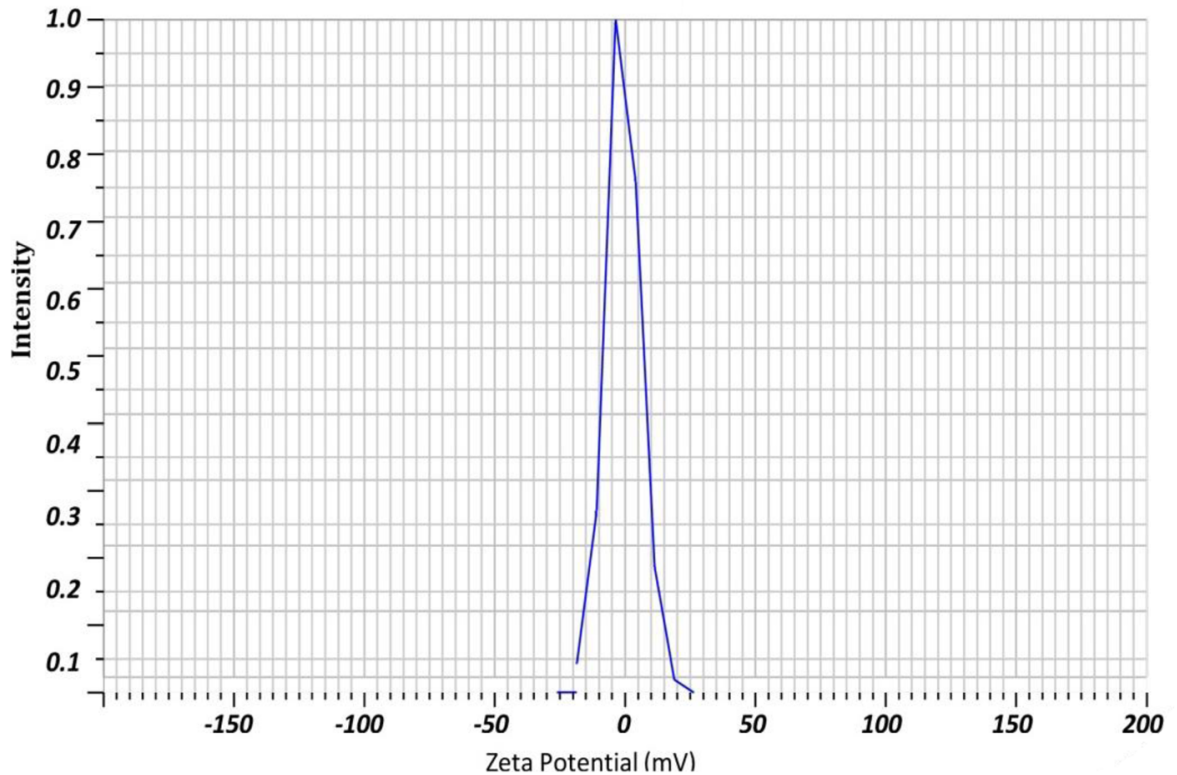

Fig. 4 a Droplet size of optimized formulation. $\mathbf{b}$ Zeta potential of optimized formulation

percent drug content $=99.51$ to $99.70, \mathrm{pH}=5.2$ to 5.4 , transparency $=$ good, and phase separation $=$ no, and creaming was observed after 3 months. Optimized emulgel preparation was stable and consistent for all evaluated parameters.

\section{Statistical analysis}

One-way ANOVA method was used for statistical analysis of different formulations, when the $P$ value $\leq 0.05$ than the values of all formulations were observed significant statistically. $P$ values of different parameters were observed as $0.0010,0.0021,0.0350$, and 0.0073 for zeta potential, droplet size, percent drug release, and percent PDI respectively. Linearity was observed for all responses at $\leq 0.05 P$ value. So it was obvious that the obtained data was significant.

\section{Discussion}

Nanoemulgel was used as a carrier for transdermal delivery of primaquine due to its permeation enhancing properties. With the help of transdermal delivery of drugs, we minimized the problems associated with 


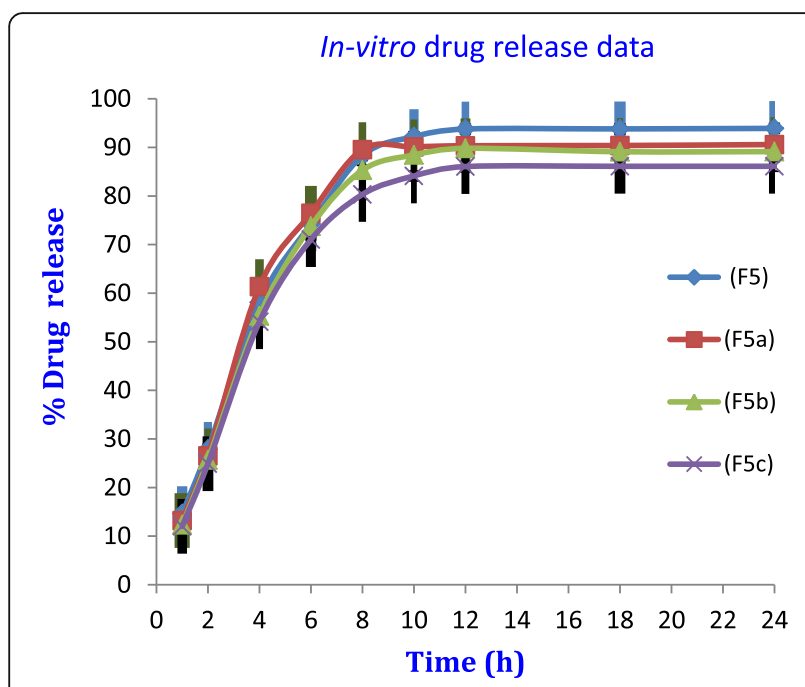

Fig. 5 In vitro drug release profile for optimized nanoemulsion (F5) and nanoemulgel formulation ( $F 5_{a}, F 5_{b}$, and $\left.F 5_{c}\right)$

conventional dosage form of primaquine. The nanoemulgel formulation was a primaquine loaded multicomponent system having oil, $S_{\text {mix }}$, water, and HPMC as gel base. The nanoemulsions were formulated by figuring out the range of concentrations for all components. All nanoemulsion formulations (F1-F9) were optimized for droplet size, zeta potential, percent PDI, and percent drug release. The TEM images of nanoemulsion showed spherical structure, and uniformity of droplet size revealed by low PDI value. The conductivity test confirmed that nanoemulsion was o/w type. The values of viscosities were observed to be low which did not indicate its applicability onto the skin. Thus, F5 formulation showed the $68.5 \mathrm{~nm}$ droplet size and its $-0.7 \mathrm{mv}$ zeta potential value indicated that it created sufficient repulsive forces between the droplets. The F5 formulation

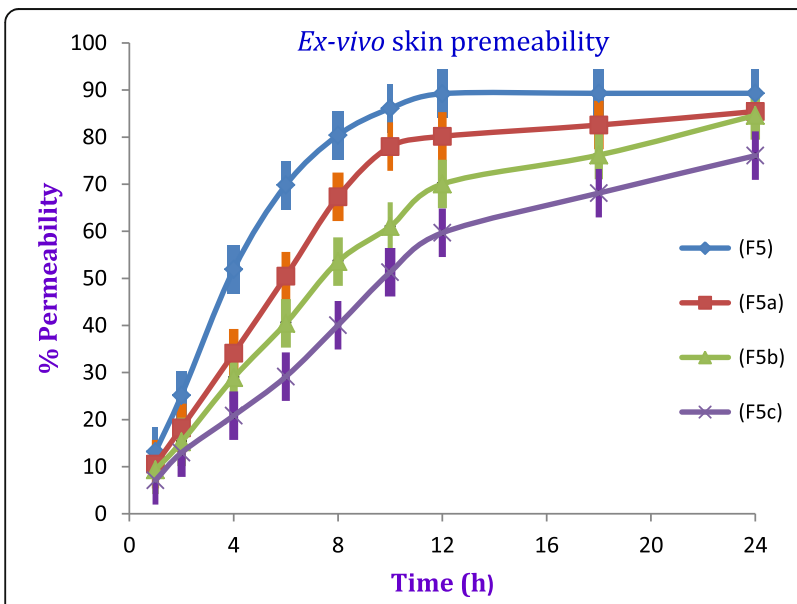

Fig. 6 Ex vivo skin percent permeability for optimized nanoemulsion (F5) and nanoemulgel formulations ( $F 5_{a}, F 5_{b}$, and $\left.F 5_{c}\right)$ showed the highest (91.28\%) percent drug release after $5 \mathrm{~h}$ (Table 2). The conductivity of F5 formulation was maximum $(126.6 \mathrm{mS} / \mathrm{cm})$ which indicated that it has sufficient concentration of water which facilitates its initial permeability; based on these considerations, F5 formulation was selected for loading in the hydrogel. When zeta potential, droplet size, percent drug release, and percent PDI were compared through one-way ANOVA for all formulations, then $P$ values observed as 0.001, $0.0021,0.0350$, and 0.0073 respectively. The $P$ values for these responses were observed lower than 0.05 which showed that the model was significant. So it was obvious that the obtained data was significant. Thus, F5 nanoemulsion was incorporated in the hydrogel containing HPMC K15M (1-2\%). The $\mathrm{pH}$ of all nanoemulgel preparations was near to skin $\mathrm{pH}(5.5 \mathrm{pH})$ so it was suitable for transdermal application without causing irritancy. The viscosity was increased with HPMC K15M concentration. As the variable 1 concentration was increased from low level to high level (5-15\%) and variable 2 concentrations were decreased from high level to low level (45-35\%), then permeability was increased. The increased permeability may be due to castor oil permeation in the lipid bilayer of stratum corneum. The Transcutol $\mathrm{P}$ acted as a permeation enhancer. Ex vivo skin flux study of optimized $\mathrm{F}_{\mathrm{b}}$ emulgel formulation showed the permeabilty of drug into specific diameter of skin. Zero-order release was followed by the nanoemulgel formulation and it was revealed through data fitting in various models. The value of correlation coefficient was higher for zero-order model than other models. So it was obvious that formulation followed zero-order kinetic. The prepared nanoemulgel was observed to possess good permeability without the addition of any permeability enhancers, although the efficacy of the nanoemulgel system was depending upon its composition (castor oil, $\mathrm{S}_{\text {mix }}$, and hydrogel). The thermodynamic stability was also evaluated for nanoemulgel preparation; and during the study, there were no changes observed in $\mathrm{pH}$ and transparency. Thus, primaquine loaded emulgel preparation could be beneficial to avoid adverse effects of the conventional dosage form.

\section{Conclusion}

The nanoemulsion loaded hydrogel of primaquine with optimum viscosity was prepared for transdermal application. Nanoemulgel was prepared by using HPMC K15M into nanoemulsion because HPMC K15M was responsible for significant viscosity. The penetration rate of nanoemulgel was greater than other drug solutions. The great penetration rate was achieved by the incorporation of Transcutol P as a cosurfactant. The optimized formulation was justified by using statistics. Stability studies 
confirmed nanoemulgel to be a promising carrier for the delivery of primaquine.

\section{Abbreviations}

$S_{\text {mix }}$ : Surfactant and cosurfactant mixture; HPMC: Hydroxy propyl methyl cellulose; TEM: Transmission electron microscopy; PDI: Poly dispersity index; ANOVA: Analysis of variance; SD: Standard deviation; IAEC: Institutional Animal Ethical Committee; CPCSEA: Committee for Purpose of Control and Supervision of Experiments on Animals

\section{Acknowledgements}

The authors express special thanks to Suman Jain (Director, School of Studies in Pharmaceutical Sciences, Jiwaji University, Gwalior) and Navneet Garun for their kind support (Department of Pharmaceutics, School of Studies in Pharmaceutical Sciences, Jiwaji University, Gwalior).

\section{Authors' contributions}

PS designed and optimized the study and developed the methodology. PS performed the experiments, collection, and interpretation data. PS wrote the manuscript. MT contributed to manuscript revision and provided supervision. PS and MT read and approved the final manuscript.

\section{Funding}

This work did not receive any fund from any source.

\section{Availability of data and materials}

The datasets of research were collected from experiments and analysis of variables during the current study. These datasets are available from the corresponding author on reasonable request.

\section{Ethics approval and consent to participate}

The ex vivo skin permeation study work was completed at School of Studies in Pharmaceutical Sciences, Jiwaji University, Gwalior, according to the protocols permitted by the Committee for Purpose of Control and Supervision of Experiments on Animals (CPCSEA), Ministry of Social Justice and Empowerment, Government of India, under the reference no. IAEC/JU/ 47 on the recommendations of the Institutional Animal Ethical Committee of Jiwaji University (Gwalior, India).

\section{Consent for publication}

Not applicable.

\section{Competing interests}

No competing interests to declare.

Received: 24 February 2020 Accepted: 13 May 2020

Published online: 23 June 2020

References

1. Thiagarajan P, Ravi PU (2011) Nanoemulsions for drug delivery through different routes. Res in Biotech 2(3):1-13

2. Chen H, Khemtong C, Yang X, Chang X, Gao J (2011) Nanonization strategies for poorly water-soluble drugs. Drug Discov Today 16(7-8):354360

3. Garala KC, Shinde AJ, Shah PH (2009) Formulation and in-vitro characterization of monolithic matrix transdermal systems using HPMC/ Eudragit S100 polymer blends. Int J Pharmacy Pharm Sci 1(1):108-120

4. Ambade KW, Jadhav SL, Gambhire MN, Kurmi SD, Kadam VJ, Jadhav KR (2008) Formulation and evaluation of flurbiprofen microemulsion. Curr Drug Deliv 5(1):32-41

5. Yilmaz E, Borchert $H$ (2006) Effect of lipid-containing, positively charged nanoemulsions on skin hydration, elasticity and erythema-an in-vivo study. Int J of Pharm 307(2):232-238

6. Date AA, Joshi MD, Patravale VB (2007) Parasitic diseases: liposomes and polymeric nanoparticles versus lipid nanoparticles. Adv Drug Deliv Rev 59(6):505-521

7. Singh KK, Vingkar SK (2008) Formulation, antimalarial activity and biodistribution of oral lipid nanoemulsion of primaquine. Int J of Pharm 347: $136-143$
8. Mihaly GW, Ward SA, Edwards G, Nicholl DD, Orme ML, Breckenridge AM (1985) Pharmacokinetics of primaquine in man. I. Studies of the absolute bioavailability and effects of dose size. Br J Clin Pharmacol 19(6):745-750

9. Ward SA, Mihaly GW, Edwards G, Looareensuwan S, Phillips RE, Chanthavanich P, Warrell DA, Orme ML, Breckenridge AM (1985) Pharmacokinetics of primaquine in man. II. Comparison of acute vs chronic dosage in Thai subjects. Br J Clin Pharmacol 19(6):751-755

10. Price AH, Fletcher KA (1986) The metabolism and toxicity of primaquine Prog Clin Biol Res 214:261-278

11. Sharma P, Tailang M (2018) In-vivo study of orodispersible tablet of primaquine. Int J of Pharm Sci and Res 9(8):3506-3510

12. Chen X, Chang X, Du D, Li J, Xu H, Yang X (2006) Microemulsion-based hydrogel formulation of ibuprofen for topical delivery. Int J of Pharm 315(12):52-58

13. Chudasama A, Patel V, Nivsarkar M, Vasu K, Shishoo C (2011) Investigation of microemulsion system for transdermal delivery of itraconazole. J Adv Pharm Technol Res 2:30-38

14. Garala K, Patel J (2011) Design and development of a self nanoemulsifying drug delivery system for telmisartan for oral drug delivery. J Pharm Invest 1 : $112-118$

15. Lee EA, Balakrishnan P, Kil C, Song CK, Choi JH, Noh GY, Park CG, Choi AJ, Chung SJ, Shim CK, Kim DD (2010) Microemulsion based hydrogel formulation of itraconazole for topical delivery. J Pharm Invest 40:305-311

16. Gohel MC, Nagori SA (2010) Fabrication and evaluation of hydrogel thickened microemulsion of ibuprofen for topical delivery. Indian J Pharm Educ Res 44:189-196

17. Bagley DM, Gardner JR, Holland G, Lewis RW, Regnier JF, Stringer DA, Walker AP (1996) Skin irritation: Reference chemicals data bank. Toxicol In Vitro 10:1-6

18. Abdul-Rasool BK, Abu-Gharbieh EF, Sahar A, Fahmy SA, Saad HS, Khan SA (2010) Development and evaluation of ibuprofen transdermal gel formulations. Trop J Pharm Res 9:355-363

19. Baboota S, Shakeel F, Ahuja A, Ali J, Shafiq S (2007) Design, development and evaluation of novel nanoemulsion formulations for transdermal potential of celecoxib. Acta Pharm 57(3):315-332

20. Suppaibulsuk B, Prasassarakich P, Rempel GL (2010) Factorial design for nanosized polyisoprene synthesis via differential microemulsion polymerization. Polym Adv Technol 21:467-475

21. Aboofazeli R, Barlow DJ, Lawrence MJ (2000) Particle size analysis of concentrated phospholipid microemulsions: I. Total intensity light scattering. AAPS PharmSci 2(2):1-13

22. Biruss B, Kahlig H, Valenta C (2007) Evaluation of an eucalyptus oil containing topical drug delivery system for selected steroid hormones. Int $J$ of Pharm 328(2):142-151

23. Mou D, Chen H, Du D (2008) Hydrogel-thickened nanoemulsion system for topical delivery of lipophilic drugs. Int J of Pharm 353(1-2):270-276

\section{Publisher's Note}

Springer Nature remains neutral with regard to jurisdictional claims in published maps and institutional affiliations.

\section{Submit your manuscript to a SpringerOpen ${ }^{\circ}$ journal and benefit from:}

- Convenient online submission

- Rigorous peer review

- Open access: articles freely available online

High visibility within the field

Retaining the copyright to your article

Submit your next manuscript at $>$ springeropen.com 